\title{
Analysis of Common Problems of Noise and Vibration in Building Heating Ventilation Air Conditioning Design
}

\author{
XiaolongRuan ${ }^{1, *}$, Yisong Yin ${ }^{2}$ \\ ${ }^{1}$ Chongqing Information Technology Design Co., Ltd. Chongqing 400000, China \\ ${ }^{2}$ Shanghai Fengsi Chengjie Architectural Design and Research Institute Co., Ltd. Shanghai 201900, China
}

\begin{abstract}
With the continuous growth of my country's economy and the continuous improvement of the people's economic level, the demand for the design of heating, ventilation and air conditioning systems has also increased. The HVAC system is very important in the building environment. When optimizing the relevant design, it is necessary to always consider the design details of the HVAC system and adjust the integrity of the design, especially to take preventive measures in areas where noise and vibration may occur to avoid heating serious problems caused by noise and general vibration when the communication system is running. This article outlines how to avoid and control common noise and vibration problems when designing HVAC projects.
\end{abstract}

\section{Introduction}

The building's HVAC system can manually or intelligently control the comfort of the human living environment in the building. The equipment of the HVAC system generates a certain amount of vibration and noise due to the mechanical operation during operation, which affects the comfort of human living and the structural stability of the building. In building structure design, the main design types are divided into seismic design, durability design and fatigue design. In the design process of prevention and control of common problems in buildings, the main common problem is vibration. The shape and frequency of vibration generated by equipment and human activities in the building will damage the building. The design of the HVAC system fully considers the structure of the building, the characteristics of the equipment, the frequency of use of the equipment, and the form and stability of vibration. These are the main sources of noise and vibration in the design process and are necessary to ensure the safety of the system. At the same time, it provides people with a comfortable living environment.

\section{Analysis of HVAC design concept}

When implementing a specific architecture design, it must be combined with actual conditions. HVAC design is an important part of the entire building design. In this case, the quality of the HVAC system design will inevitably affect the entire building. According to the functional analysis of the HVAC system project, three main aspects are considered: heating, ventilation and air conditioning. Since the HVAC system project is implemented in a specific process, it involves many very important parts, such as thermodynamics and electrical engineering. Therefore, when planning the structure of the air conditioning system, it is necessary to combine reality to maximize the basic goals of heating, ventilation and air conditioning [1].

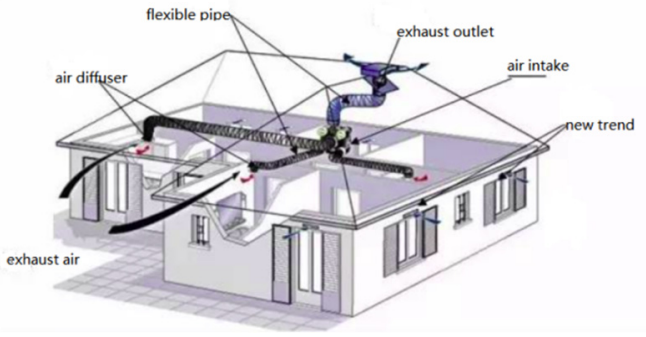

Figure 1. HVAC system design

\section{Probe into the hazards of noise and vibration in building HVAC engineering}

The noise and vibration of the building's HVAC system are mainly caused by air conditioning terminals, such as air conditioning units, circulating water pumps, cooling towers, fans, and air conditioning window cabinets. These types of equipment are prone to noise and vibration during use. When the impact of noise and equipment vibration in decibels exceeds the allowable limit, it will affect people's comfort, and the noise and vibration of the building's heating, ventilation and air-conditioning system will have a significant impact on people's production and life. At the same time, continuous vibration can cause permanent damage to the equipment, reduce the efficiency of the equipment, and shorten its service life. In addition, the noise and vibration of the building's HVAC system will increase 
the cost of subsequent maintenance. Therefore, the personnel involved in the design and construction should pay close attention to the noise and vibration of the building's HVAC system [2].

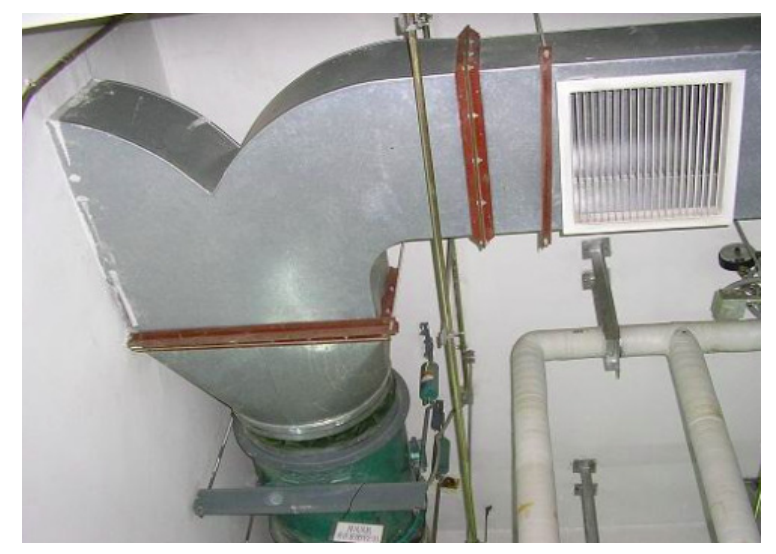

Figure 2. Noise in building HVAC engineering

\section{Common problems such as noise and vibration in building HVAC design and their causes}

\subsection{The sound transmission problem of the exhaust vent}

Generally, when designing and configuring the building's HVAC system, it is usually necessary to install indoor air conditioners. Most HVAC equipment is mainly designed for household use, so it is relatively slow and diffusers can be installed to improve heat dissipation. If the HVAC device is equipped with a diffuser, it will generate a relatively large amount of noise. Based on years of professional experience of relevant personnel, we know that the noise generated by air conditioners is relatively low and will not have a significant impact on the lives of residents. However, if the installation location is near the nozzle, the noise will continue to increase due to the influence of the airflow, and may exceed a reasonable range. There is no noise reduction and noise reduction equipment in the design of the HVAC system, the noise cannot be effectively controlled, and the noise level continues to rise, which eventually affects people's daily life and exceeds its tolerance limit.

\subsection{The effect of holes and gaps on noise}

All types of equipment have some common problems, such as noise and vibration in HVAC configurations, but this is not the only equipment that affects noise. Holes and gaps are also important noise sources. Generally, the smaller the holes and gaps, the better the sound insulation effect and the lower the noise. On the contrary, the larger the holes and gaps, the worse the sound insulation effect and the greater the noise. Secondly, the location of the holes and gaps will also affect the sound insulation effect and noise level. Improper location of the holes and gaps makes it impossible to select points at the cutting edges of the two walls, which may result in poor sound insulation.

\subsection{Problems with exhaust facilities}

Smoke exhaust fans are usually used in buildings to ensure the circulation of outdoor air and indoor air. They are very important exhaust systems. However, during normal operation of the exhaust facility, noise problems will occur, and the increase in its operating speed will increase the efficiency of air circulation and make the corresponding noise problems more serious. Because the blades of the exhaust fan are relatively close, the problem of smoke exhaust noise during normal operation increases, which affects the lives of residents [3].

\subsection{Problems in the design of the air supply system}

In some buildings that require high-quality environments, there are dedicated personnel who need to provide users and residents (such as conference rooms and star hotels) with a relatively quiet and comfortable environment, and use devices for the visually impaired to keep the rooms quiet. However, when the muffler unit is installed outside the air circulation hole, if the exact location of the muffler unit is not correctly determined, the muffler unit will be destroyed and the indoor noise situation will change. A detailed analysis of the air supply system in some meeting rooms shows that the air supply system in the meeting room mainly uses a silent exhaust system. In this exhaust mode, noise is mainly transmitted to certain equipment and structures in the air recirculation process, and then to the control room of the computer room. As a result, the noise is transmitted to the room through the equipment, making the power distribution system of the conference room A lot of noise is generated.

\subsection{Noise problem of air supply equipment}

The HVAC design in many buildings includes adding noise suppression devices during the installation of the air supply system to reduce the noise generated during the air supply. However, the attenuation process of the ventilation duct is ignored, and noise cannot be avoided even if there is a muffler in the air duct. Similarly, many methods of unlocking devices are useless. One of the disadvantages is that some air enters other devices and the noise will be amplified.

\subsection{The noise problem caused by the air-conditioned machine room close to the conference room.}

Some meeting rooms use low-speed HVAC equipment, but the noise is still loud, which may directly affect normal use. This is because the building next to the meeting room is equipped with an air-conditioned room, and there is an exhaust pipe leading to the engine room on the top of the meeting room, and there is a hole in the wall. Since the opening is completely covered, noise from the 
computer room will seep into the wall, making the conference room too noisy. In addition, some air conditioners have poor vibration damping functions, and the generated vibration and noise are transmitted to the conference room through the holes.

\subsection{Noise problem of cooling tower}

Compared with other equipment, the cooling tower can make the air-conditioning system run smoothly, but it has some shortcomings that cannot be ignored, such as noise pollution and long working hours, which greatly affect people's quality of life. With the continuous improvement of people's living standards, people have begun to improve the working conditions of air conditioners, which is especially reflected in the installation of cooling towers. When planning the HVAC system, the designer needs to consider the installation of the cooling tower, as well as the specific conditions of the building, as well as the needs of people. It is also necessary to consider the relationship between the cooling tower and the surrounding area, provide enough airflow to attack the tower, and stabilize the pressure level.

\section{Specific measures to solve the noise and vibration problems in the design of building HVAC engineering}

\subsection{Treatment measures in the design of exhaust vents}

When designing a building's public HVAC system, installing slow-speed HVAC equipment or diffusers above it will produce a certain degree of noise. Taking into account the noise problem of the exhaust outlet, employees must comprehensively and objectively analyze the causes of noise, select appropriate noise suppression devices, and control the noise level within a limited standard range. For example, the hotel lobby is equipped with slow and decentralized air conditioning. In addition, a centralized ventilation valve design is used on the side wall. The ventilation space in the lobby is about 4 square meters, the lobby is about 2 square meters, the round-trip wind speed is $2 \mathrm{~m} / \mathrm{s}$, and the wind speed noise can be clearly heard. The main reason for the noise is that the HVAC system is too close to the exhaust pipe, and the parties have not taken appropriate disposal measures. With this in mind, employees should apply a layer of sound insulation material on the outside of the return air duct to prevent noise from being transmitted through the room's return air duct. The muffler is connected to the exit of the hall, effectively reducing the noise level in decibels. Install sound insulation materials on the exhaust holes, and use sound insulation materials to reduce noise decibels. In addition, cotton can also be buried in construction pits to suppress noise and provide people with a quiet living environment [4].

\subsection{Effectively control the noise source of noise and vibration}

When creating a HVAC project, the first step to effectively avoiding common problems (such as noise and vibration) is to effectively manage the sources of noise and vibration. Only by controlling the noise source can the problem of noise and vibration be completely solved. Effective control of the noise source requires a thorough understanding of the noise source, otherwise the noise source cannot be effectively controlled if the noise source is not found. Therefore, HVAC system designers are improving the research on noise sources in order to obtain sufficient knowledge before design, and make full use of relevant knowledge in the actual design process, and determine the exact cause of noise and vibration. Secondly, after the noise source is successfully detected, it is necessary to effectively control the noise source to minimize the occurrence of noise, or take scientific and reasonable measures to eliminate noise.

\subsection{Scientific selection of system equipment}

When selecting equipment for building ventilation systems, relevant departments and employees should pay attention to the following points: (1) According to the size and ventilation requirements of the building, select equipment with appropriate air volume to avoid excessive air volume in the system. (2) Selecting the fan needs to be debugged. Through the test, we know how much noise the fan will make when it is running, and based on this, we have eliminated some unacceptable equipment noises. Generally, when selecting equipment, the principles of low noise and high efficiency should be followed. It is recommended to tilt the fan blades backward. (3) When designing the building's HVAC system, it is important to ensure that the actual airflow in the duct meets the acceptable building noise requirements. (4) When adjusting the position of the inlet and outlet of the fan, please do not set it to sudden change. At the same time, designers can use 100 to $150 \mathrm{~mm}$ flexible connectors for shock absorption. When selecting and using dust-proof materials, it is important to choose synthetic leather and fiberglass that are as fireproof as possible.

\subsection{Reasonable choice of muffler}

Muffler is an important equipment to solve the noise problem of modern buildings. Therefore, when designing the building's HVAC system, the designer must accurately determine the function of the muffler, and choose a muffler device with more appropriate model parameters according to the actual building conditions. Ideally, noise and vibration can be reduced. Especially when selecting and using the muffler, the designer firstly calculates the noise suppression level according to the internal structure and ventilation requirements of the building, and then accurately calculates the noise suppression level according to the determined value. It is necessary to select the appropriate muffler model and characteristics. The analysis of previous work experience concluded that the 
typical noise frequencies in modern buildings are $125 \mathrm{~Hz}$, $250 \mathrm{~Hz}$, and $500 \mathrm{~Hz}$. Therefore, it is relatively recommended to choose low and intermediate frequency resistors or resonance mufflers. In addition to considering the actual sound absorption capacity of the muffler, the wind speed of the muffler must also be calculated and analyzed. Under normal circumstances, the wind speed of the muffler should not exceed $6 \mathrm{~m} / \mathrm{s}$. If the muffler is installed in the indoor pipeline, you must also ensure that the wind speed of the muffler does not exceed $5 \mathrm{~m} / \mathrm{s}$, usually you need to choose a middle/low frequency resistor or a resonance muffler. Designers can also customize the detailed information during a specific design process, for example: operate the muffler in the form of an oval or sickle-shaped sound-absorbing blade to reduce noise.

\subsection{Treatment measures in the design of the airrsupply system}

According to usage requirements, the meeting room is equipped with an air supply system and a silencer. However, when the silencer was installed in the ventilation hole of the HVAC system, the noise level (decibel) exceeded the limit. Today, most buildings use built-in air conditioners to increase the frequency of noise to some extent. If the noise cancellation device is only installed in certain positions, the overall effect of noise cancellation is not ideal. System inspections show that the building's HVAC equipment uses exhaust methods instead of pipes. When the air returns, some of the air enters the control room of the computer room, the device captures the sound, and then the duct is used as a medium to guide the room. In order to deal with this situation, designers must install mufflers according to standard specifications. In addition, strictly control the working wind speed of the air conditioner to make the noise and noise reduction effect reach the expected level [5].

\subsection{Noise prevention measures for cooling towers}

There are two types of cooling tower noise: fan noise and dripping noise. Designers can install a muffler manifold at the outlet of the fan, and a waterproof glass wool board is attached to the elbow to make it have good waterproof performance and effectively reduce fan noise. By using sound insulation materials or quiet cooling towers and using noise isolation devices to protect one side of the residential area, the noise caused by falling water can be effectively reduced. Therefore, when choosing a cooling tower, the cooling tower must be placed correctly, and sound insulation devices must be used to reduce noise. The closer the obstacle is to the sound source, the better the sound insulation effect. When designing heating, ventilation, and air-conditioning systems, problems such as noise and vibration are often encountered. These problems do not occur alone, but are spread through various media, which not only affect the normal use of buildings, but also affect people's lives and physical health.
At present, people do not pay special attention to noise and vibration when designing heating, ventilation and air-conditioning systems. This problem is only discovered when the building is completed and reconstruction has a significant impact on the environment [6]. Therefore, designers must better understand the noise and vibration in the HVAC design process, formulate effective prevention and control plans to prevent excessive vibration and noise, and strictly abide by the rules related to HVAC building design to improve HVAC design.

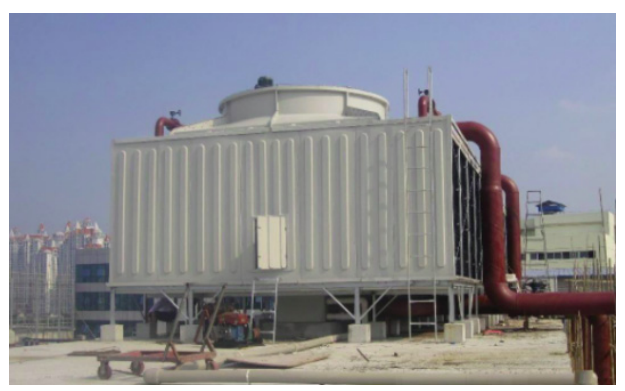

Figure 3. Low noise cooling tower

\subsection{Preventive measures against noise generated by the air-conditioning machine room close to the conference room}

The noise reduction design needs to be strengthened near the air-conditioning machine room, especially near the meeting room. On the basis of concrete, cork blocks were installed as the main anti-vibration means, and springs were installed near the air-conditioning machine room to prevent vibration caused by two-level vibration and vibration of sound insulation technology. At the same time, the power socket on the top of the conference room must be cut off to effectively reduce the vibration and noise of the HVAC unit and computer room, and prepare for the quiet environment of the conference room.

\section{Conclusion}

In summary, the design of heating, ventilation and air-conditioning systems is a very important part of the construction process of modern building projects and can have a positive impact on people's daily lives. However, during the design and installation of the HVAC project, many parts have noise and vibration problems. These problems are not only manifested, but also spread through various media, and may have serious consequences for human health. Therefore, in reduction measures in time to control noise and vibration problems as effectively as possible.

\section{References}

1. PanpanLiu. Prevention of common problems of noise and vibration in building HVAC design[J]. Ju She, 2021(05): 80-81+99.

2. LnlanSong. Research on the prevention and treatment of common noise and vibration in building 
HVAC design[J]. Jiangxi Building Materials, 2020(08): 64-65.

3. FengliangWang. Analysis of common problems such as noise and vibration in building HVAC design[J]. Urban Construction Theoretical Research (Electronic Edition), 2020(14):43.

4. YangLiu. Analysis of common problems such as noise and vibration in building HVAC design[J]. Ju She, 2020(05): 96.

5. KewenXin, WenhuLii. Prevention of common problems of noise and vibration in building HVAC design[J]. Information Weekly, 2020, 000(001): P.1-1.

6. Lanlan Song. Research on the prevention and treatment of common noise and vibration in building HVAC design[J]. Jiangxi Building Materials, 2020, No.259(08):70-71vibration in building HVAC design[J]. Ju She, 2021(05): 80-81+99.

7. LnlanSong. Research on the prevention and treatment of common noise and vibration in building HVAC design[J]. Jiangxi Building Materials, 2020(08): 64-65.

8. FengliangWang. Analysis of common problems such as noise and vibration in building HVAC design[J]. Urban Construction Theoretical Research (Electronic Edition), 2020(14):43. 